\title{
EFFECTS OF EXERCISE ON APPETITE-REEULATING HORMONES, PERCEIVED HUNGER, AND ENERGY INTAKE: A NARRRATIVE OVERVIEW
}

\author{
Marsa Gleason, ${ }^{1, A, B, C, D}$ Basil H. Aboul-Enein, ${ }^{2, A, B, C, D}$ Josh Bernstein,, ${ }^{3, D}$ Joanna Kruk4, D \\ ${ }^{1}$ California University of Pennsylvania, Department of Exercise Science \& Health Promotion, California, USA \\ ${ }^{2}$ London School of Hygiene \& Tropical Medicine, Department of Global Health \& Development, London, United Kingdom \\ ${ }^{3}$ A.T. Still University of Health Sciences, College of Graduate Health Studies, Kirksville, USA \\ ${ }^{4}$ University of Szczecin, Faculty of Physical Education and Health Promotion, Szczecin, Poland

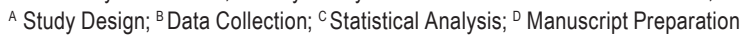

\author{
Address for corpespondence: \\ Joanna Kruk \\ University of Szczecin \\ Faculty of Physical Education and Health Promotion \\ Cukrowa 12, 71-004 Szczecin, Poland \\ E-mail: joanna.kruk@univ.szczecin.pl
}

\begin{abstract}
Ahstract Controlling appetite, perceived hunger, and energy intake are important factors in weight management. This narrative review examines the effect of different forms, intensities and duration of exercise on the appetite-regulating hormones leptin, acylated ghrelin, glucagon like peptide-1 (GLP-1) and peptide tyrosine tyrosine $3-36\left(\mathrm{PYY}_{3-36}\right)$, perceptions of hunger, and energy intake in overweight/obese, and normal weight populations. The studies reviewed compared exercise intensities- low, moderate, and high intensity, and modes of exercise- aerobic and resistance training. The studies selected in this narrative review included participants that ranged in age from 13-57 years old, male and female, previously sedentary and physically active, and normal weight and overweight/obese individuals- defined by body mass index standards (BMI). The primary benefits of exercise on appetite regulation are seen with moderate to high-intensity aerobic exercise; with the most notable relative energy deficit resulting from an exercise bout (at any intensity) that expends the most energy. Further research is warranted to determine if there exists a tendency to overcompensate for energy expended during exercise in certain populations.
\end{abstract}

Key Worls Exercise, Appetite regulation, Hunger, Weight management

\section{Introduction}

Creating an energy deficit is essential for weight loss. However, increasing exercise and therefore, energy expenditure, does not always result in desired weight loss. In a recent review, Caudwell, Gibbons, Finlayson, Naslund, and Blundell (2013) reported that, although the research predominantly shows an acute bout of exercise does not create an increase in hunger, desire to eat, or energy intake, when exercise is extended over a few days and caloric intake is monitored for a longer period, partial compensation for increased energy expenditure can occur. In these cases, even though individuals are increasing physical activity, they are subsequently increasing 
caloric intake thereby undermining their own weight loss efforts. High-intensity exercise is correlated with a transient reduction in hunger that may be due to a temporary decrease in the active component of the appetitestimulating hormone ghrelin (Caudwell et al., 2013). The appetite-regulating hormones acylated ghrelin, leptin, GLP-1, and PYY $Y_{3-36}$ are recognized to be a major influence on energy homeostasis (Klok, Jakobsdottir, Drent, 2007). Ghrelin has an appetite stimulating effect, whereas increases in leptin, GLP-1, and PYY $3-36$ tend to have appetite-suppressing effects, except in cases of leptin resistance in which increased levels of leptin do not correlate with appetite-suppression. These hormones respond differently to varying exercise modes (cardiovascular and resistance training), intensities, and duration (Caudwell et al., 2013; Klok et al., 2007). The purpose of this narrative review is to examine and describe the current evidence on the effect different exercise forms (cardiovascular and resistance training), intensities, and duration of exercise has on appetite-regulating hormones (leptin, acylated ghrelin, GLP-1, and PYY $3-36$ ), perceptions of hunger, and energy intake in overweight/obese, and normal weight populations.

\section{Methodls}

Nine academic electronic databases were used in the narrative search: PubMed; Scopus; ProQuest; EBSCOhost; SpringerLink; ArticleFirst; Taylor \& Francis; Wiley Online; and ScienceDirect. The academic databases were selected based of their academic rigor, aim, biomedical scope and accessibility. A combination of search terms included 'exercise, 'physical activity', high intensity exercise', 'low intensity exercise', strength training', 'gender', 'appetite-regulating hormones, 'hunger', 'energy intake', 'overweight', obesity', 'weight management', 'leptin', 'acylated ghrelin', 'GLP-1', and 'PYY $3-36$ '. References from retrieved articles were reviewed to identify additional relevant publications for the years 2007 through October 2017. The results generated by the search were limited to the English language. Publications were reviewed for relevance to the topic. References from retrieved articles were reviewed to identify additional applicable publications.

\section{Disculssion}

\section{Low-Intensity Aerobic Exercise}

In researching the effects of low-intensity exercise on appetite-regulating hormones, King, Wasse, Broom, and Stensel (2010) found that although a 60-minute brisk walk in healthy adult males resulted in a moderate energy deficit, it had no effect on appetite, acylated ghrelin, or energy intake. Similar results were found for healthy adults cycling for a short duration (30-minutes) at a low-intensity (below the aerobic-anaerobic threshold); the exercise had no effect on hunger/satiety sensations or on subsequent energy intake (Erdmann, Tahbaz, Lippl, Wagenpfeil, Schusdziarra, 2007). Low-intensity exercise, such as walking, gentle yoga, tai chi, and stretching have many positive health benefits including modest energy expenditure which can aid in weight management; however, it is unlikely to result in the appetite-reducing effects that have been seen with higher-intensity exercise.

\section{Moderate and High-Intensity Aerobic Exercise}

Moderate and high-intensity exercise has appetite reducing effects that may be beneficial for those looking to improve body composition. High-intensity exercise has a more notable acute-effect on appetite suppression than moderate-intensity exercise; however, the effects on appetite may be just as notable with moderate-intensity exercise 
in the hours after. In a comparison of high-intensity cycling bouts (10, 4-min cycling bouts at $\left.85-90 \% \mathrm{VO}_{2} \mathrm{max}\right)$, to steady-state moderate aerobic exercise (60-min cycling at $60 \% \mathrm{VO}_{2} \mathrm{max}$ ), there was greater appetite suppression during the high-intensity cycling (Deighton, Karra, Batterham, Stensel, 2013); the authors, however, found that both exercise intensities suppressed appetite over the 8-hour trial, with no statistically significant differences. There was an increase in $\mathrm{PYY}_{3-36}$ immediately after the steady-state exercise, however $\mathrm{PYY}_{3-36}$ was higher in the hours after high-intensity training. Despite the minor differences in $\mathrm{PY}_{3-36}$, both exercise intensities were effective at inducing an energy deficit without compensation of energy intake following exercise. Similar results were found in physically active women; Howe et al. (2016) compared isocaloric bouts (expending about $500 \mathrm{kcal}$ per exercise session) of moderate-intensity $\left(60 \% \mathrm{VO}_{2} \max \right)$ and high-intensity $\left(85 \% \mathrm{VO}_{2} \max \right)$ exercise. Although there were no differences in appetite hormones following exercise between-high and moderate intensities, there was a significant main effect for time. Immediately after exercise GLP-1 and PYY ${ }_{3-36}$ were significantly higher than at baseline (before exercise), and ghrelin was significantly lower; however, 60-minutes after exercise all three hormones were at levels close to baseline. Appetite ratings were higher at 60 -minutes post-exercise with both exercise intensities with no significant differences.

In trained long-distance male runners, there were surprisingly no changes to PYY immediately after a 78-minute run at $78 \%$ HRmax, the authors suggest this may be a result of an overnight fast prior to exercise; whereas in previous studies showing a rise in PYY the participants consumed a pre-exercise meal (Kojima, Ishibashi, Ebi, Goto, 2016). Despite no significant changes in PYY, the trained long-distance runners did report significantly lower appetite ratings and had significantly lower absolute energy intake immediately after exercise (and up to 60-minutes post-exercise), compared to a non-exercise day (Kojima et al., 2016).

These results show that despite the immediate appetite-suppressing effects of an exercise, the energy expended during the exercise session had a more notable effect on relative energy intake (calories expended during the exercise minus the calories consumed after the exercise). Deighton, Barry, Connon, and Stensel (2013) compared sprint intervals (6, 30-second Wingate tests) to a 60-minute bout of moderate-intensity steadystate cycling (at $68 \% \mathrm{VO}_{2} \max$ ) and found that although acylated ghrelin was lowest during and after the sprintintervals, the relative energy intake was lower with the endurance exercise trial. This was due to the greater energy expenditure during the longer bout of steady-state cycling without subsequent overcompensation of energy intake following the exercise session.

Douglas et al. (2015) found that two consecutive days of moderate-intensity exercise (60-minute treadmill run at $70 \%$ of $\mathrm{VO}_{2}$ peak) in healthy-young men resulted in a significant suppression of perceived hunger and significantly elevated PYY over both days of the exercise trial when compared to 2-days of no exercise; there was also significantly lower relative energy intake over the 2-days of exercise compared to the non-exercise days.

\section{Resistance Training}

There has been limited research into the potential effects of resistance training on appetite-regulating hormones, hunger, and subsequent energy intake. Guelfi, Donges, and Duffield (2013) compared the effects of 12-weeks of moderate-intensity aerobic exercise to resistance training and found an increase in perceived fullness following a meal in the aerobic exercise group with no other statistically significant differences between hunger ratings or appetite-regulating hormones. According to Broom et al. (2009), resistance exercise suppresses hunger to up to one hour compared with no exercise; however, this response is less influential than aerobic exercise. 
In a subsequent study, Laan, Leidy, Lim, and Wayne (2010) looked at the meal energy intake following resistance exercise, compared to aerobic exercise, and found no differences in energy intake during the meal following exercise. The aerobic exercise, however, resulted in greater energy expenditure, and therefore a lower energy intake relative to energy expended in the meal following exercise. The resistance exercise did have a relative energy intake lower compared to no exercise. These results suggest that the amount of energy expended during exercise is more significant, for weight loss purposes, than the effects of appetite regulating hormones of either aerobic or resistance training (Lann et al., 2010). Aerobic exercise, which can be sustained for an extended period-of-time, typically results in greater energy expenditure; this, in addition to the appetite-suppressing effects immediately postmoderate/high intensity aerobic exercise, provide a better option for reducing relative energy intake for individuals trying to lose weight.

\section{Gender Differenences}

There are varying results when it comes to the appetite-suppressing effects of exercise between genders. Hagobian et al. (2009) found differences in appetite-regulating hormones between men and women after moderateintensity exercise (50-65\% $\mathrm{VO}_{2}$ max until $30 \%$ of total daily energy expenditure expended). The comparisons were made in overweight men and women, and found that for women only, acylated ghrelin levels, in addition to appetite ratings, were increased after exercise. The authors suggested their findings may help explain why when previously sedentary men and women follow exercise training programs with ad libitum feeding, men typically lose body fat, but women do not (Hagobian et al., 2009). In contrast, Hagobian et al. (2013) compared the effects of exercise and appetite control in normal weight, active men and women and found acute exercise (cycling at $70 \% \mathrm{VO}_{2} \max$ until $30 \%$ of total daily energy expenditure was expended) effectively suppresses short-term appetite regardless of gender. Overweight and obese women may not benefit from the appetite-suppressing effects of low to moderateintensity exercise when compared to normal weight individuals and overweight men. What was noteworthy between the two studies is the level of exercise intensity; in the study showing increased hunger and ghrelin levels after overweight women exercised (Hagobian et al., 2009), the exercise intensity was set between $50-65 \% \mathrm{VO}_{2}$ max. When compared to Hagobian et al., 2013 in which the exercise intensity was consistent moderate-intensity at $70 \% \mathrm{VO}_{2} \mathrm{max}$, it is possible that an exercise intensity fluctuating between low to moderate-intensity (Hagobian et al., 2009) is not sufficient to produce any appetite-suppressing effects. According to Vantansever-Ozen, TiryakiSonmez, Bugdayci, and Ozen (2011), a minimum exercise intensity of at least $60 \% \mathrm{VO}_{2}$ max is likely necessary to have any effect on appetite-regulating hormones.

There is insufficient evidence to suggest women are disadvantaged when using exercise to suppress appetitehormones and perceived hunger after exercise at an intensity of at least $60 \% \mathrm{VO}_{2} \max$. It is important to note that both referenced studies comparing men and women controlled for the menstrual phase of the women participating; women participated during the early follicular phase of menstruation (1-4 days after menstruation). It is generally accepted that women tend to increase their energy intake during the luteal phase of menstruation compared to the follicular phase (Campolier et al., 2016), therefore if not controlled for, menstruation could confound the results of a study comparing appetite hormones in women. 


\section{Overweight/Obesity, Exercise, and Appetite Regulation}

Individuals looking to lose weight stand to gain major benefits from the potential effect exercise has on appetite-regulating hormones. When overweight/obese individuals, male and female, exercise at intensities above $60 \% \mathrm{VO}_{2}$ max, there are beneficial short- and long-term changes to appetite-regulating hormones, perceived hunger, and relative energy intake. Martins et al. (2015) found obese individuals to have appetite-suppressing benefits of moderate-and high-intensities, similar to that of normal weight individuals, with no significant differences between intensities. Steady-state cycling at 70\% HRmax (to expend 250 calories) had similar short-term appetitesuppressing effects as sprint-intervals which raised heart rate intermittently to $85-90 \%$ HRmax (also to expend 250 calories). There were beneficial effects of both exercise intensities on appetite-hormones, perceived hunger, and relative energy intake over non-exercise, with no statistically significant differences between moderate- or highintensity exercise. In a comparison of lean vs overweight/obese individuals exercising for 60 -minutes at moderate to low intensities ( 60 and $58 \% \mathrm{VO}_{2}$ peak respectively), there were only small differences in appetite, relative energy intake, and appetite-regulating hormone concentrations between the two groups over the 8-hour trial. Regardless of BMI, there was a suppression of appetite immediately after exercise, and an increase of PYY and GLP-1 in the hours after exercise. There were no changes to acylated ghrelin in response to exercise in either group which may be a result of the $\mathrm{VO}_{2}$ peak being too low $\left(59 \%\right.$ peak $\left.\mathrm{VO}_{2}\right)$ to elicit a change in acylated ghrelin (Douglas et al., 2017). Therefore, based on the aforementioned authors, if the exerciser has reached a moderate-high intensity there will be a short-term appetite-suppressing effect on appetite.

In a 12-week study comparing low-and high-intensity exercise in obese adolescents (13-18 years-old); both groups exercised 3 days/week expending 350 calories each exercise session. The high-intensity exercise group saw increases in $\mathrm{PYY}_{3-36}$, and reduced energy intake based on 24-hour food logs (it is important to note that energy intake was not assessed daily over the 12-weeks) (Prado et al., 2015). In addition to measuring the effects exercise has on appetite, the researchers also monitored body composition over the 12-week period. Both exercise therapies were effective at reducing body mass, BMI, fat mass, and increasing fat free mass, with no significant differences between groups. It is important to note that both groups received multidisciplinary treatment including nutrition and psychological counseling over the 12-weeks. Counseling and subsequent awareness of dietary behaviors could be an explanation for why, despite more significant appetite-suppressing effects in the high-intensity exercise group, both groups had similar improvements in body composition.

\section{Conclusions}

It is estimated that $70 \%$ of adults in the United States are overweight or obese and the prevalence of obesity among adolescents age 12-19 years old is continuing to increase with one in five adolescents considered obesethese statistics are estimated from 2013-2014 National Health and Nutrition Examination Survey (Fryar, Carrol, Ogden, 2016). Overweight and obesity increases the risk of developing chronic health diseases such as diabetes and heart disease which is good reason for a growing interest in studying the role that appetite-regulating hormones such as leptin, ghrelin, and $\mathrm{PYY}_{3-36}$, have on overeating, and subsequently weight management. The studies reviewed suggest a positive correlation between moderate to high-intensity exercise and appetite suppression through changes in appetite-regulating hormones, perceived hunger, and relative energy intake. 
In this narrative review, exercise intensities were differentiated based on levels of maximal oxygen uptake $\left(\mathrm{VO}_{2}\right.$ max) or maximum heart rate ( $\mathrm{HRmax}$ ) with low-intensity exercise at a $\mathrm{VO}_{2}$ max below $60 \%$, moderate-intensity exercise $\mathrm{V} \mathrm{VO}_{2}$ max or $\mathrm{HRmax}$ between $60-85 \%$, and high-intensity exercise a $\mathrm{VO}_{2}$ max or $\mathrm{HRmax} 85 \%$ and above. The studies selected in this review included participants that ranged in age from 13-57 years old, male and female, previously sedentary and physically active, and normal weight and overweight/obese individuals- defined by body mass index standards (BMI). Many of the studies included in this review measured perceived hunger which was assessed using $100 \mathrm{~m}$ visual analogue scales with descriptors anchored at each end; the assessment calculated an overall appetite score to include hunger, satisfaction, fullness, and prospective food consumption.

Low-intensity exercise, below $60 \% \mathrm{VO}_{2}$ max, has little-to-no effect on appetite-regulating hormones, perceived hunger or energy intake; although it does create a modest energy deficit (King et al., 2010) that would likely benefit those making an-effort to lose weight. Moderate and high-intensity aerobic exercise above $60 \% \mathrm{VO}_{2} \mathrm{max}$ - whether steady-state or sprint-intervals - show the most promise in suppressing appetite (Howe et al., 2016). Resistance exercise has some short-term appetite-reducing effects (Broom et al., 2009); however, the longer-term effects are not well studied, and aerobic exercise (steady-state or sprint-intervals) is more likely to create a relative energy deficit; an important factor in weight reduction.

There were varying results in comparing exercise and appetite-suppression between genders; overweight/ obese women may not have similar benefits of appetite suppression with exercise compared to overweight/obese men. Although these differences were only found with exercise intensity of 50-65\% (Hagobian et al., 2009), an exercise intensity of at least $60 \% \mathrm{VO}_{2}$ max is likely necessary to have any effect on appetite-regulating hormones (Vantansever-Ozen et al., 2011).

Further research is needed to determine the effects of exercise on appetite in overweight/obese women, including variations between phases of the menstrual cycle. Overweight/obese individuals, who stand to gain the most from any changes that exercise has on appetite and relative energy intake, have been shown to have positive changes to appetite with exercise. The exercise can be moderate to high intensity, as-long as it is over $60 \%$ $\mathrm{VO}_{2} \mathrm{max}$, it can be steady-state exercise or sprint-intervals. This narrative review did not find evidence to support the theory that individuals overcompensate for energy expended by consuming more after exercise; although it is important to note that the participants of all the studies reviewed were aware that they were being monitored and there is the possibility of bias affecting their energy intake following exercise. In summary, moderate to highintensity exercise affects appetite-regulating hormones and perceived hunger leading to reduced relative energy intake. Understanding how exercise affects appetite-regulating hormones will help fitness, nutrition, health, and wellness professionals tailor exercise and dietary recommendations to an individual.

\section{Clinical Implications}

For nutrition and health professionals working with overweight/obese clients; developing a regular exercise routine including moderate to high-intensity aerobic exercise should be a goal to help regulate appetite hormones, reduce perceived hunger, and create an energy deficit. For substantial health benefits, the United States Department of Health and Human Services recommend adults include physical activity in the amounts of 150-300 minutes of moderate-intensity exercise per week or 75-150 minutes of vigorous-intensity exercise per week, in addition to strength training two or more days per week; it is advised that children and adolescents do 60-minutes or more of physical activity per day (Centers for Disease Control and Prevention, 2016). Physical activity should not be 
discouraged out of a fear of energy overcompensation. For previously sedentary individuals or those with injuries limiting activity, even low-intensity exercise can create a modest energy deficit (King et al., 2010). Regulating appetite is one factor in achieving weight loss; clinicians should be aware of the potential benefits of a multidisciplinary approach to weight loss, including athletic training, nutrition counseling, and behavioral therapy.

\section{Limitations}

The focus of this review was on the potential benefits that exercise can have on appetite regulation in overweight/obese individuals. Potential negative effects of other populations of interest should be noted; athletes especially those looking to gain lean body mass should be aware of the appetite suppressing effects immediately after moderate and high-intensity aerobic exercise and resistance training given that optimal fueling for recovery includes a post-meal snack or meal within 30-minutes of exercise. Another population that should take note are those recovering from eating disorders in which appetite-suppression would not be a desired effect. Another limitation worth mentioning is that the authors did not distinguish between the hormonal and biochemical response to different kinds of exercise based on age, gender, activity, physical performance, body composition, initial hormonal status and other relevant factors. Given that narrative reviews are considered among the lowest levels of evidence-based research, tend to be mainly descriptive in nature, and can often include an element of selection bias, some level of caution should be considered regarding its conclusions.

\section{References}

Broom, D.R., Batterham, R.I., King, J.A., Stensel, D.J. (2009). Influence of resistance and aerobic exercise on hunger, circulating levels of acylated ghrelin, and peptite YY in healthy males. American Journal of Physiology-Regulatory, Integrative and Comparative Physiology, 296 (1), 29-35. DOI: 10.1152/ajpregu.90706.2008.

Campolier, M., Thondre, S.P., Clegg, M., Shafat, A., Mcintosh, A., Lightowler, H. (2016). Changes in PYY and gastric emptying across the phases of the menstrual cycle and the influence of the ovarian hormones. Appetite, 107, 106-115. DOI: 10.1016/j. appet.2016.07.027.

Caudwell, P., Gibbons, C., Finlayson, G., Naslund, E., Blundell, J. (2013). Physical activity, energy intake, and obesity: the links between exercise and appetite. Current Obesity Reports, 2 (2), 185-190. DOI: 10.1007/s13679-013-0051-1.

Centers for Disease Control and Prevention (2016). Current physical activity guidelines. Retrieved from: https://www.cdc.gov/cancer/ dcpc/prevention/policies_practices/physical_activity/guidelines.htm.

Deighton, K., Barry, R., Connon, C.E., Stensel, D.J. (2013). Appetite, gut hormone and energy intake responses to low volume sprint and traditional endurance exercise. European Journal of Applied Physiology, 113, 1147-1156. DOI: 10.1007/s00421-012-2535-1.

Deighton, K., Karra, E., Batterham, R.L., Stensel, D.J. (2013). Appetite, energy intake, and PYY3-36 responses to energy-matched continuous exercise and submaximal high-intensity exercise. Applied Physiology Nutrition and Metabolism, 38, 947-952. DOI: 10.1139/apnm-2012-0484.

Douglas, J.A., King, J.A., McFarlane, E., Baker, L., Bradley, C., Crouch, N., ..., Stensel, D.J. (2015). Appetite, appetite hormone and energy intake responses to two consecutive days of aerobic exercise in healthy young men. Appetite, 92 (1), 57-65. DOI: 10.1016/j.appet.2015.05.006.

Douglas, J.A., King, J.A., Clayton, D.J., Jackson, A.P., Sargeant, A., Thackray, E., ..., Stensel, D.J. (2017). Acute effects of exercise on appetite, ad libitum energy intake and appetite-regulatory hormones in lean and overweight/obese men and women. International Journal of Obesity, 41 (12), 1737-1744. DOI: 10.1038/ijo.2017.181.

Erdmann, J., Tahbaz, R., Lippl, F., Wagenpfeil, S., Schusdziarra, V. (2007). Plasma ghrelin levels during exercise - effects of intensity and duration. Regulatory Peptides, 143 (1-3), 127-135. DOI: 10.1016/j.regpep.2007.05.002.

Flint, A., Raben, A., Blundell, J.E., Astrup, A. (2000). Reproducibility, power and validity of visual analogue scales in assessment of appetite sensations in single test meal studies. International Journal of Obesity Related Metabolic Disorders, 24, 38-48. DOI: 10.1038/sj.ijo.0801083. 
Fryar, C.D., Carroll, M.D., Ogden, C.L. (2016). Prevalence of overweight, obesity, and extreme obesity among adults aged 20 and over: United States 1960-1962 through 2013-2014. National Center for Health Statistics Health E-Stats. Retrieved from: https://www. cdc.gov/nchs/data/hestat/obesity_adult_13_14/obesity_adult_13_14.pdf.

Guelfi, K.J., Donges, E.D., Duffield, R. (2013). Beneficial effect of 12 weeks of aerobic compared with resistance exercise training on perceived appetite in previously sedentary overweight and obese men. Metabolism, 62 (2), 235-243. DOI: 10.10.16/j. metabol.2012.08.002.

Hagobian, T.A., Sharoff, C.G., Stephens, B.R., Wade, G.N., Silva, G.E., Chipkin, S.R., Braun, B. (2009). Effects of exercise on energyregulating hormones and appetite in men and women. American Journal of Physiology-Regulatory, Integrative and Comparative Physiology, 296 (2), 233-242. DOI: 10.1152/ajpregu.90671.2008.

Hagobian, T.A., Yamashiro, M., Hinkel-Lipsker, J., Streder, K., Evero, N., Hackney, T. (2013). Effects of acute exercise on appetite hormones and ad libitum energy intake in men and women. Applied Physiology Nutrition and Metabolism, 38, 66-72. DOI: 10.1139/apnm-2012-0104.

Howe, S.M., Hand, T.M., Larson-Meyer, D.E., Austin, K.J., Alexander, B.M., Manore, M.M. (2016). No effect of exercise intensity on appetite in highly-trained endurance women. Nutrients, 8, 223. DOI: 10.3390/nu8040223.

King, J.A., Wasse, L.K., Broom, D.R., Stensel, D.J. (2010). Influence of brisk walking on appetite, energy intake, and plasma acylated ghrelin. Medicine \& Science in Sports \& Exercise, 42 (3), 485-492. DOI: 10.1249/MSS.0b013e3181ba10c4.

Klok, M.D., Jakobsdottir, S., Drent, M.L. (2007). The role of leptin and ghrelin in the regulation of food intake and body weight in humans: a review. Obesity Reviews, 8 (1), 21-34. DOI: 10.1111/j.1467-789X.2006.00270.x.

Kojima, C., Ishibashi, A., Ebi, K., Goto, K. (2016). The effect of a $20 \mathrm{~km}$ run on appetite regulation in long distance runners. Nutrients, 8 (672), 1-13. DOI: 10.3390/nu8110672.

Laan, D.J., Leidy, H.J., Lim, E., Campbell, W.W. (2010). Effects and reproducibility of aerobic and resistance exercise on appetite and energy intake in young, physically active adults. Applied Physiology, Nutrition, and Metabolism, 35, 842-847. DOI: 10.1139/ H10-072.

Martins, C., Stensvold, D., Finlayson, G., Holst, J., Wisloff, U., Kulseng, B., ..., King, N.A. (2015). Effect of moderate- and high-intensity acute exercise on appetite in obese individuals. Medicine and Science in Sports and Exercise, 47 (1), 40-48. DOI: 10.1249/ MSS. 000000000000372.

Prado, W.L., Lofrano-Prado, M.C., Cardel, M., Balagopal, P., Oyama, L.M., Praxedes-Gomes, P., ..., Hill, J.O. (2015). Effect of a 12-week low vs. high intensity aerobic exercise training on appetite-regulating hormones in obese adolescents: a randomized exercise intervention study. Pediatric Exercise Science, 27, 510-517. DOI: 10.1123/pes.2015-0018.

Vantansever-Ozen, S., Tiryaki-Sonmez, G., Bugdayci, G., Ozen, G. (2011). The effects of exercise on food intake and hunger: relationship with acylated ghrelin and leptin. Journal of Sports Science and Medicine, 10, 283-291. Retrieved from: http://www. jssm.org/gecjssm-10-283.xml.xml.

Cite this article aS: Gleason, M., Aboul-Enein, B.H., Bernstein, J., Kruk, J. (2018). Effects of Exercise on Appetite-Regulating Hormones, Perceived Hunger, and Energy Intake: A Narrative Overview. Central European Journal of Sport Sciences and Medicine, 2 (22), 5-12. DOI: 10.18276/cej.2018.2-01. 